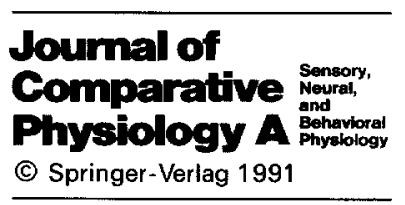

\title{
Audition in vampire bats, Desmodus rotundus
}

\author{
U. Schmidt ${ }^{1 *}$, P. Schlegel ${ }^{2}$, H. Schweizer ${ }^{2}$, and G. Neuweiler ${ }^{2}$ \\ ${ }_{1}^{1}$ Zoologisches Institut der Universität Bonn, Poppelsdorfer Schloß, W-5300 Bonn 1, Federal Republic of Germany \\ ${ }^{2}$ Zoologisches Institut der Universität München, Luisenstraße 14, W-8000 München 2, Federal Republic of Germany
}

Accepted November 9, 1990

Summary. 1. Within the tonotopic organization of the inferior colliculus two frequency ranges are well represented: a frequency range within that of the echolocation signals from 50 to $100 \mathrm{kHz}$, and a frequency band below that of the echolocation sounds, from 10 to $35 \mathrm{kHz}$. The frequency range between these two bands, from about 40 to $50 \mathrm{kHz}$ is distinctly underrepresented (Fig. 3B).

2. Units with $B F s$ in the lower frequency range $(10-25 \mathrm{kHz})$ were most sensitive with thresholds of -5 to $-11 \mathrm{~dB}$ SPL, and units with BFs within the frequency range of the echolocation signals had minimal thresholds around $0 \mathrm{~dB}$ SPL (Fig. 1).

3. In the medial part of the rostral inferior colliculus units were encountered which preferentially or exclusively responded to noise stimuli. - Seven neurons were found which were only excited by human breathing noises and not by pure tones, frequency modulated signals or various noise bands. These neurons were considered as a subspeciality of the larger sample of noisesensitive neurons. - The maximal auditory sensitivity in the frequency range below that of echolocation, and the conspicuous existence of noise and breathing-noise sensitive units in the inferior colliculus are discussed in context with the foraging behavior of vampire bats.

Key words: Hearing - Chiroptera - Desmodus Inferior colliculus - Tonotopy

\section{Introduction}

Among mammals, echolocating bats are the order that exploits the widest variety of food resources. Bats capture flying insects, arthropods living on vegetation, insects and fishes at the surface of water, ground-dwelling

Abbreviations: $B F$ Best frequency; $I C$ inferior colliculus

* To whom offprint requests should be sent small vertebrates, and also fruits, nectar and pollen. The most specialized of all mammals are the echolocating vampire bats of the neotropics which exclusively subsist on blood from mammals and birds (review see Schmidt 1978; Greenhall and Schmidt 1988).

Studies on audition of echolocating bats have demonstrated that the auditory system is intricately adapted to the specific requirements of species-specific acoustic foraging strategies (review see Neuweiler 1990). For instance, rhinolophids, hipposiderids and Pteronotus parnelli, which pursue flying insects in or around dense vegetation, have evolved a sophisticated cochlear fovea and neuronal specializations which allow the selective detection of wing-beating targets among echo-clutter (echo-glint detection, Schnitzler et al. 1983). In these bats the tonotopic organization of the ascending auditory pathway is heavily biased in favor of the species-specific and even individual, specific narrow frequency band used for glint detection in echolocation.

Other bat species have other specific types of auditory adaptations. For example, Plecotus auritus, Megaderma lyra and Macroderma gigas forage on prey living on substrates and their auditory systems are extremely sensitive (up to $-27 \mathrm{~dB}$ SPL) to a frequency range $(15-25 \mathrm{kHz})$ just below that of their echolocation signals (review see Neuweiler 1990). The Indian false vampire Megaderma lyra, has a hunting style similar to that of owls (Payne 1971) in that they can precisely locate and attack a source of noise in a familiar room in complete darkness (Fiedler 1979). In contrast, the false vampire does not detect prey which does not generate noise (Marimuthu and Neuweiler 1987). The auditory system is specifically adapted for detecting faint noises; these adaptations include unusually large pinnae, and auditory neurons in the lateral and central part of the inferior colliculus (IC) which specifically respond to faint noises and react poorly to other types of auditory stimuli (Rübsamen et al. 1988).

Megaderma lyra differs from horseshoe bats, in that frequencies below the band used for echolocation occupy more neural space in the inferior colliculus than do the 
frequencies of the echo signals. Thus, the auditory system of Megaderma lyra appears to be differentiated for passive acoustical prey detection in addition to echolocation.

Vampire bats depend on a nightly blood meal, and therefore have to detect and locate their prey. In a natural tropical habitat without anthropogenic blood sources such as farm stock living on pastures, the vampire's prey usually rest under cover. It is unlikely that echolocation would be of much help for detecting mammals under cover. However, if vampire bats had such extreme auditory sensitivity for faint noises as has been demonstrated in false vampires, they might be able to detect the sources for a blood meal by noises generated by the prey. Since the false vampires of the Old World and the vampires of the New World are unrelated families, possible convergent auditory adaptations would demonstrate the adaptive power of the mammalian auditory system to external evolutionary pressure.

In a first investigation of specific auditory capacities in the vampire bat, Desmodus rotundus, we looked for frequency ranges of behavioral significance as seen by overpresentation of certain frequency bands within the tonotopic organization of the IC, and we also searched for neurons that were specifically sensitive to noise.

\section{Material and methods}

The animals used in these experiments were 9 adult male Desmodus rotundus which were born and brought up in the vampire colony of the Zoological Institute, University of Bonn.

Surgery and electrophysiological methods were the same as described by Pollak and Schuller (1981) and by Rübsamen et al. (1988). Briefly, surgery was performed under halothane anesthesia (dosage 1.5 vol\%). The head was fixed in a head holder by a metal bolt glued to the frontal skull, and a hole $(1 \mathrm{~mm} \varnothing)$ was drilled into the bone covering the inferior colliculus. This opening for electrode penetrations was filled with paraffin oil and the wound margins were treated with a local anesthetic (novocain).

Recordings in awake specimens started one day after surgery and continued over $2-3$ consecutive days. Recording was through glass micropipettes, filled with $3 \mathrm{M} \mathrm{KCl}$ and a resistance of 1-2 M $\Omega$ for multi-unit, and 4-8 M $\Omega$ for single-unit recordings. The electrodes were advanced dorsoventrally through the inferior colliculus. The last track of a series of parallel penetrations was marked with two iontophoretic HRP-injections $(1 \mu \mathrm{A}, 2.5 \mathrm{~min})$ at different depths. The stereotaxic coordinates of the penetrations were reconstructed according to histological data from parasagittal and frontal sections of the brains.

BFs and thresholds were determined audiovisually, and PST histograms ( 32 sweeps, bin width $1 \mathrm{~ms}$ ) were sampled with a computer (PDP11/40).

The acoustical search stimuli consisted of pure tones $(3.5 \mathrm{~ms}$ duration, incl. $0.5 \mathrm{~ms}$ rise/fall time) and bandpassed noise $(80 \mathrm{~ms}$, $0.5 \mathrm{~ms}$ rise/fall time). In some cases frequency modulated downward sweeps and harmonic signals were presented. The loudspeakers, a condenser loudspeaker for frequencies from 15 to $120 \mathrm{kHz}$ and a dynamic one for frequencies below $15 \mathrm{kHz}$, were mounted on a hoop and placed in front of the contralateral ear. The position of the ultrasonic loudspeaker was adjusted in azimuth and elevation for the best neuronal response (Schlegel et al. 1988). The frequency characteristics of the speakers were calibrated by a 1/4" and a $1 / 8^{\prime \prime}$ B\&K condenser microphone (models 4135/4138). Stimulus intensity is expressed in $\mathrm{dB}$ SPL (re $20 \mu \mathrm{Pa}$ ).

\section{Results}

\section{Audiogram and tuning}

In 62 penetrations in the $\mathrm{IC}, \mathrm{BFs}$ and thresholds of multi-units were recorded every $50 \mu \mathrm{m}$ in 3 bats, and every $100 \mu \mathrm{m}$ in 6 bats. The BFs ranged from $800 \mathrm{~Hz}$ to about $110 \mathrm{kHz}$ (Fig. 1). The lowest thresholds of -5 to $-11 \mathrm{~dB}$ SPL were found for BFs between 10 and $25 \mathrm{kHz}$, i.e. below the frequency range of the echolocation sounds. Within the frequency range from $50-95 \mathrm{kHz}$, units were less sensitive and only reached minimal values slightly above $0 \mathrm{~dB}$ SPL. Minimal thresholds rapidly increased for BFs below $10 \mathrm{kHz}$ and above $96 \mathrm{kHz}$. At $\mathrm{BFs}$ of 2 and $100 \mathrm{kHz}$ lowest thresholds range between 20 and $30 \mathrm{~dB}$ SPL.

Pure tone thresholds were determined for 132 single units. The data confirm the results of the multi-unit recordings: the most sensitive units have $\mathrm{BFs}$ around $20 \mathrm{kHz}$, and for BFs in the ultrasonic range thresholds are above $0 \mathrm{~dB}$ SPL. The lowest BF was found at $700 \mathrm{~Hz}$ (thresholds $68 \mathrm{~dB}$ SPL).

Figure 2 demonstrates some typical tuning curves of single units. Most units were rather narrowly tuned, and some of them had upper thresholds. A few neurons responded to a wide range of frequencies, for instance one neuron with a $\mathrm{BF}$ of $65 \mathrm{kHz}$ responded $10 \mathrm{~dB}$ above threshold to pure tones from 11 to $93 \mathrm{kHz}$.

Most $Q_{10 \mathrm{~dB}}$-values were below 15. Only in the frequency range of the second harmonic of echolocation calls $(45-70 \mathrm{kHz})$ were 5 units found with $\mathrm{Q}_{10 \mathrm{~dB}}$-values above 15 (the highest $\mathrm{Q}_{10 \mathrm{~dB}}$-value was 77 at $54 \mathrm{kHz}$ ).

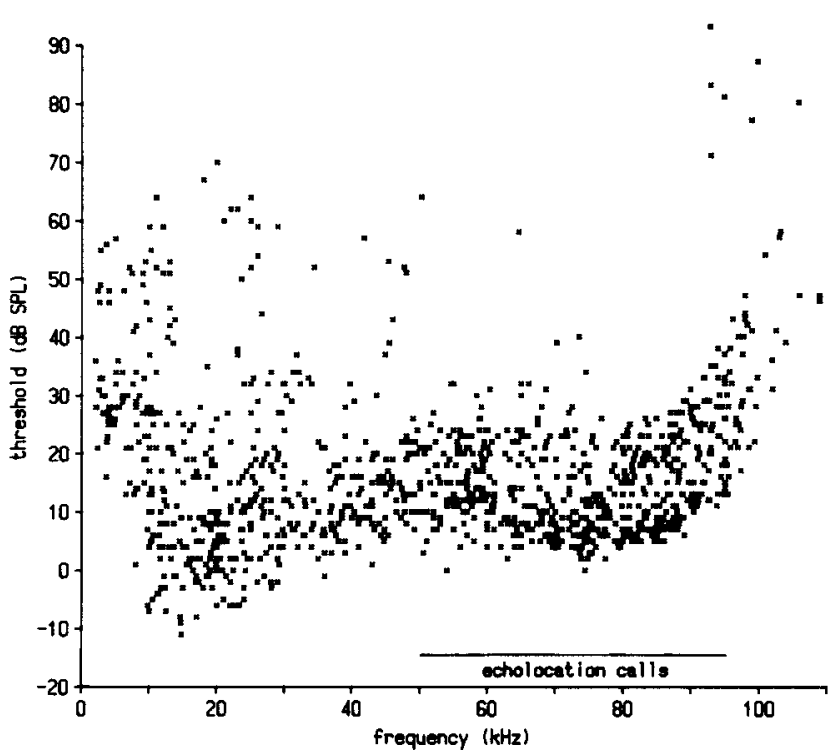

Fig. 1. Neural audiogram of Desmodus rotundus, obtained by multiunit thresholds in the IC (each dot signifies a threshold value as a function of the respective $\mathrm{BF}$ at a given electrode position). The frequency range of the echolocation calls is indicated 

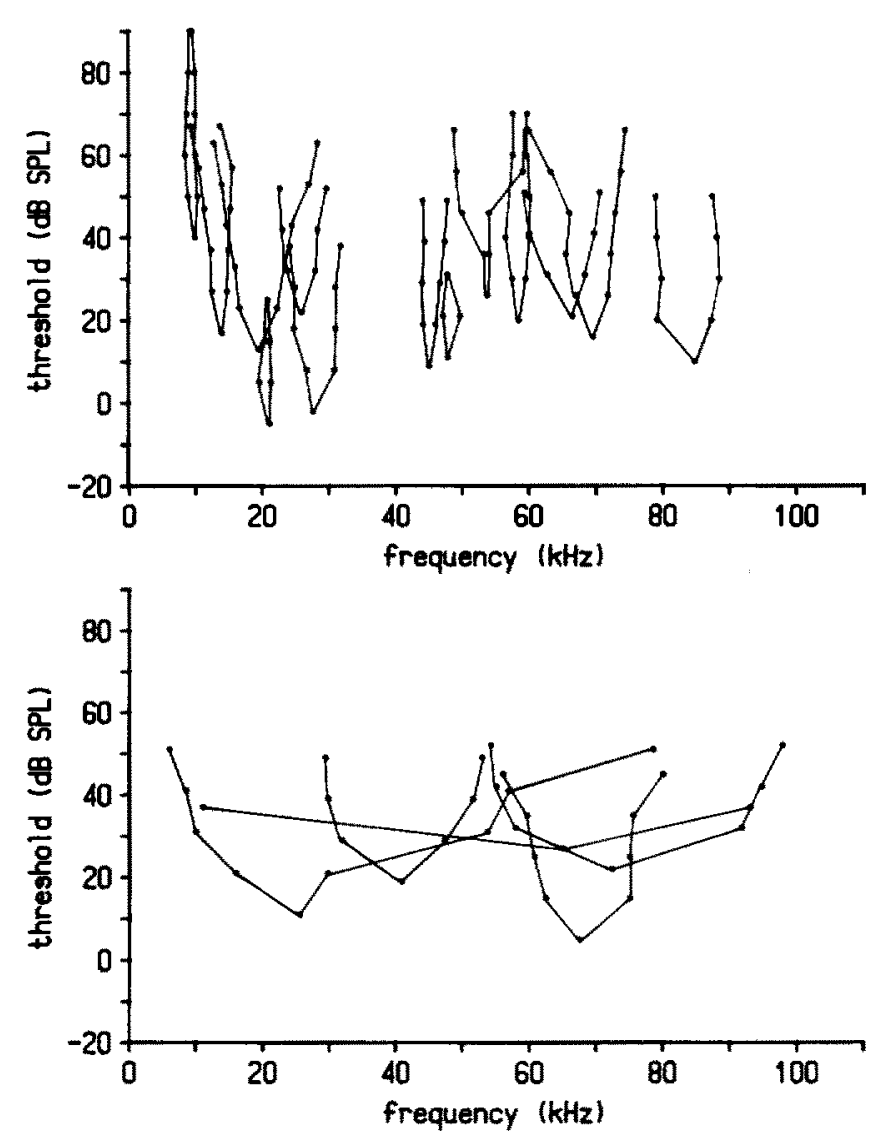

Fig. 2. Some representative tuning curves of single units (above: narrow-band units; below: broad-band units)

\section{Tonotopy}

The IC in Desmodus rotundus is covered by the cerebellum dorsally and extends about $3100 \mu \mathrm{m}$ in dorsoventral, $2800 \mu \mathrm{m}$ in lateromedial, and $1400 \mu \mathrm{m}$ in caudofrontal direction. The tonotopy of the IC was derived from 55 electrode penetrations, of which 13 were marked by HRP. Figure 3A shows the location of these penetrations through the IC.

The general tonotopic arrangement of the IC in Des modus rotundus follows the typical mammalian pattern, i.e. low frequencies are represented dorsally, and higher frequencies in progressively more ventral parts. In the posterior and medial parts of the IC the isofrequency contours follow the curved surface of the IC and fall off from the medial to the lateral margin (Fig. 3B). In the rostral part of the IC the isofrequency contours also descend medially from the top of the IC.

Two frequency ranges are well represented within the IC: frequencies from the lower limit of sensitivity to about $35 \mathrm{kHz}$ in the dorsal third, and the frequencies from about 60 to $100 \mathrm{kHz}$ in the ventral half of the IC. This latter, high frequency range does not extend to the lateral margin in the caudal third of the IC. Units with BFs between about 40 and $50 \mathrm{kHz}$ form a relatively thin slab sandwiched between the low and high frequency representations (Fig. 3B).

\section{Noise-sensitive units}

During the multi-unit recordings single units were occasionally encountered that vigorously responded to the

\section{A}

$$
\text { post }-330 \mu m \quad 330 \mu m-660 \mu m \quad 660 \mu m-990 \mu m \quad 990 \mu m-\text { ant. }
$$
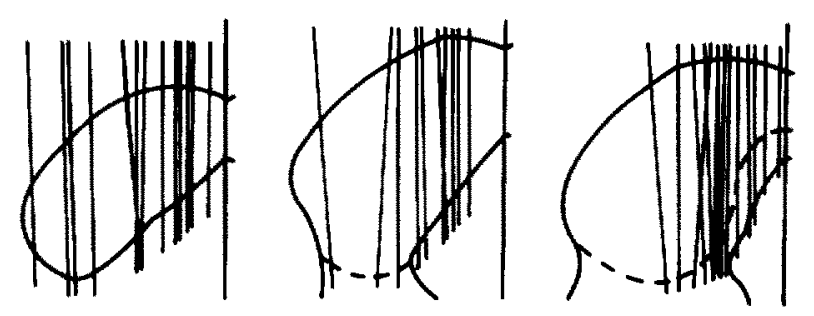
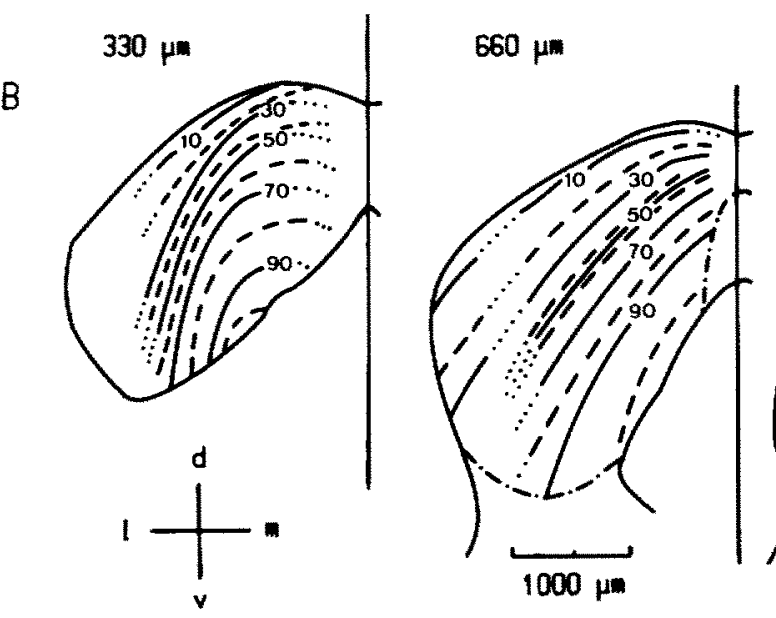

$990 \mu m$

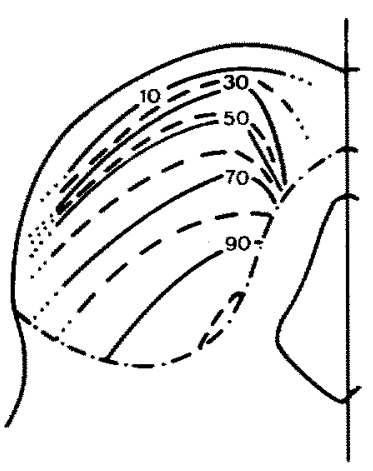

Fig. 3A. Projection of the electrode penetrations $(n=46)$, considered for establishing the tonotopy, onto 4 transverse sections of the IC (combined from 9 bats). The figures indicate the collicular range of the penetrations ( 9 additional penetrations in the medial area are not drawn). B Reconstruction of tonotopy in 3 transverse sections of the IC in $D$. rotundus (left: caudal, right: rostral). The

measurements above the drawings give the distance of the sections from the posterior margin; numbers inside the drawing delineate the particular iso-frequency layer (d: dorsal, $v$ : ventral, $l$ : lateral, $m$ : medial) 

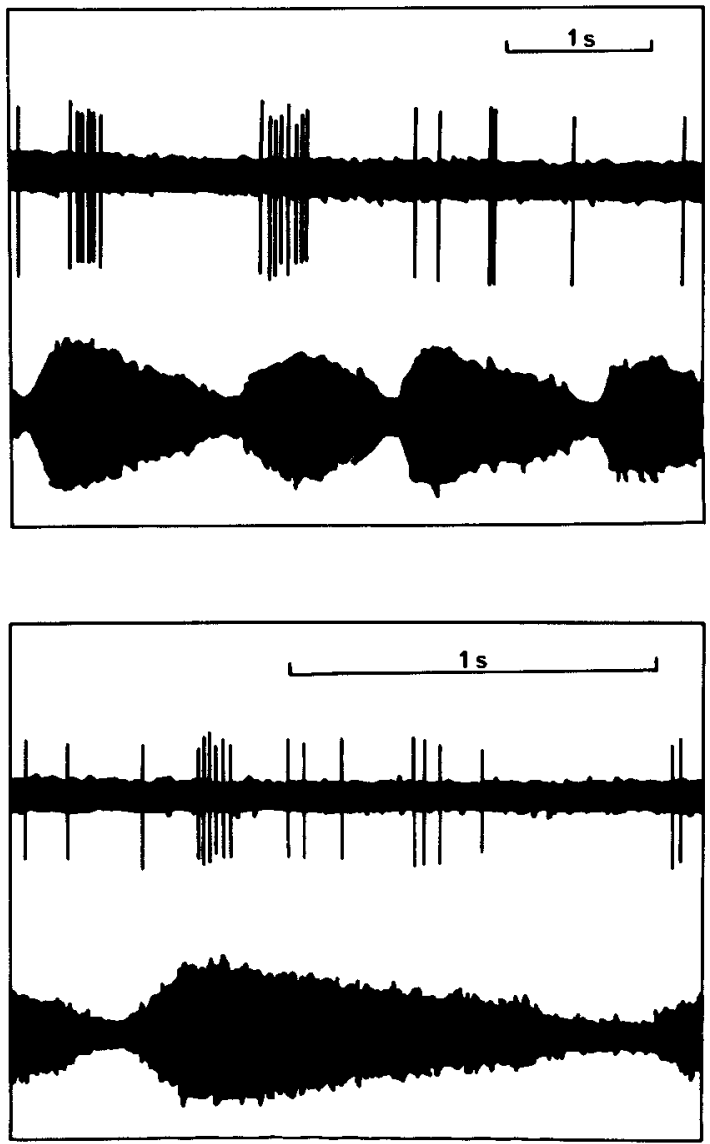

Fig. 4. Response of 2 noise-sensitive units to the playback of human respiration (intensity of noise at the head position of the bat : $30 \mathrm{~dB}$ SPL RMS; horizontal bar: $1 \mathrm{~s}$ )

faint breathing noise of the experimenters. Spike bursts locked to the breathing rhythm were also elicited when the person was $6 \mathrm{~m}$ away from the open recording chamber which housed the bat. Therefore, in 5 specimens of Desmodus rotundus we systematically searched for such units.

Single units sensitive to breathing noise were only found in the dorsal region of the IC where BFs between 10 and $30 \mathrm{kHz}$ are represented. They were most abundant in the medial part of the rostral IC where the isofrequency slabs descend ventrally, and none were encountered in the lateral areas.

Forty-six neurons were recorded which responded to human breathing noise. Seven of these units were stimulated by tape-recorded breathing noises and their responses were audiovisually assessed (Fig. 4). Another 7 units of the total sample only responded to the breathing noise and were not excited by pure tones, FM sweeps or various noise bands.

Most units $(n=32)$ sensitive to breathing noise also vigorously reacted to faint bandpass filtered noise in the frequency range below $30 \mathrm{kHz}$, and most of them $(n=28)$ also responded to more intense pure tones. Thresholds were measured for noise signals with a bandwidth of
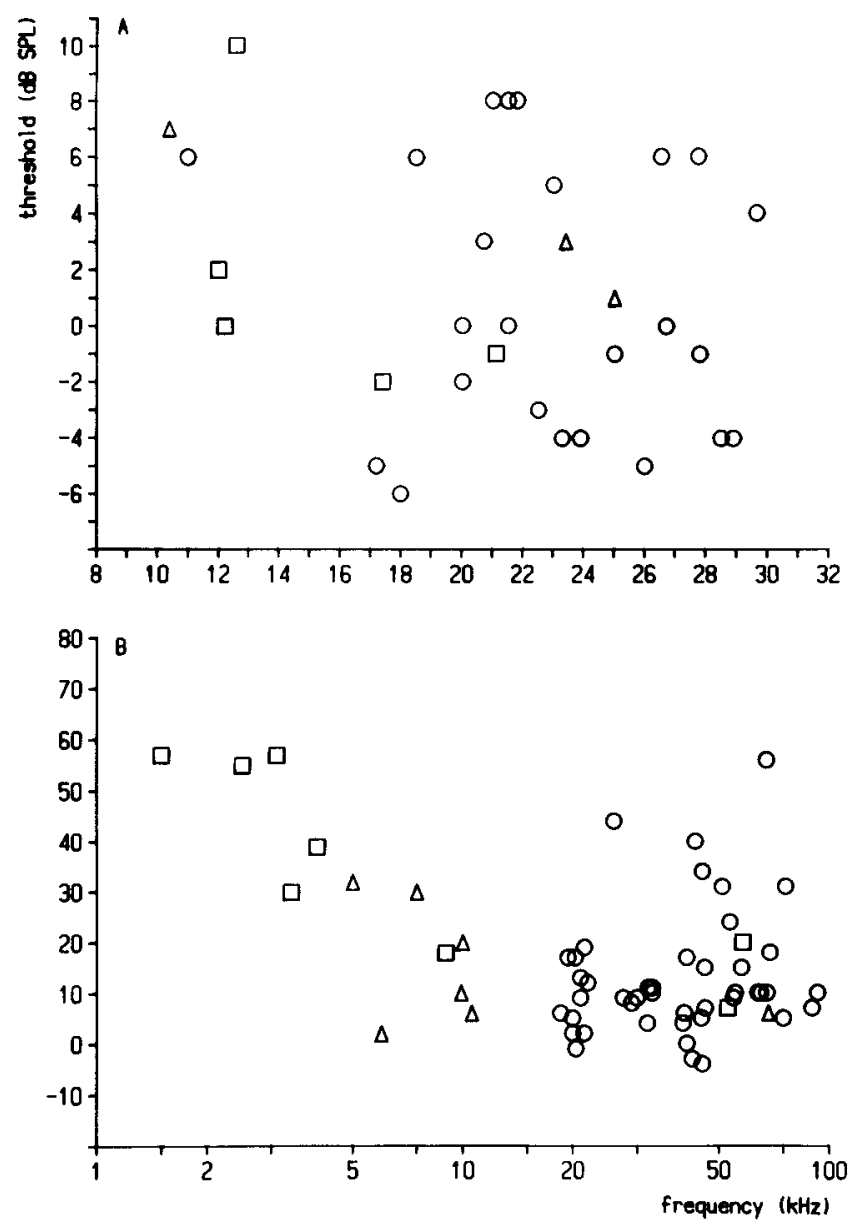

Fig. 5A. Noise-thresholds (center-frequency) of IC units $(n=32)$ sensitive to human respiration sounds. B Noise thresholds of single units ( $n=59)$ not responding to human respiration sounds (circles: bandpassed noise $\pm 10 \mathrm{kHz}$, triangles: $\pm 5 \mathrm{kHz}$, squares: $\pm 2 \mathrm{kHz}$ )

$\pm 10 \mathrm{kHz}$, and the center frequency was adjusted to the minimal threshold. As shown in Fig. 5, all units sensitive to breathing noise had minimal noise thresholds for center frequencies between 10 and $30 \mathrm{kHz}$. Noise thresholds were low and ranged from +10 to $-6 \mathrm{~dB}$ SPL. Eight units were more sensitive to narrower noise bands of \pm 5 and $\pm 2 \mathrm{kHz}$ (Fig. 5A). Thresholds to pure tones at $\mathrm{BF}$-frequency exceeded those to noise by from 10 to more than $40 \mathrm{~dB}$ (Fig. 6). Whereas there seemed to be no habituation to the breathing noise in any of the breathing sensitive neurons, some of them habituated rapidly when stimulated with band passed noise.

In 25 units sensitive to breathing noise, 3 different response patterns were recorded by PST-histograms. Fifteen units showed phasic and phasic-tonic responses during noise stimulation, and the spike rate increased monotonically with noise intensity (Fig. 7). In six units an additional strong off-response appeared when the noise intensity increased (Fig. 8). Interestingly, the magnitude of the off-response increased with stimulus duration, e.g. in one unit a $5 \mathrm{~ms}$ noise pulse elicited one off-burst, and a $80 \mathrm{~ms}$ noise pulse three off-bursts (Fig. 8C).

Four units were excited only within a narrow inten- 


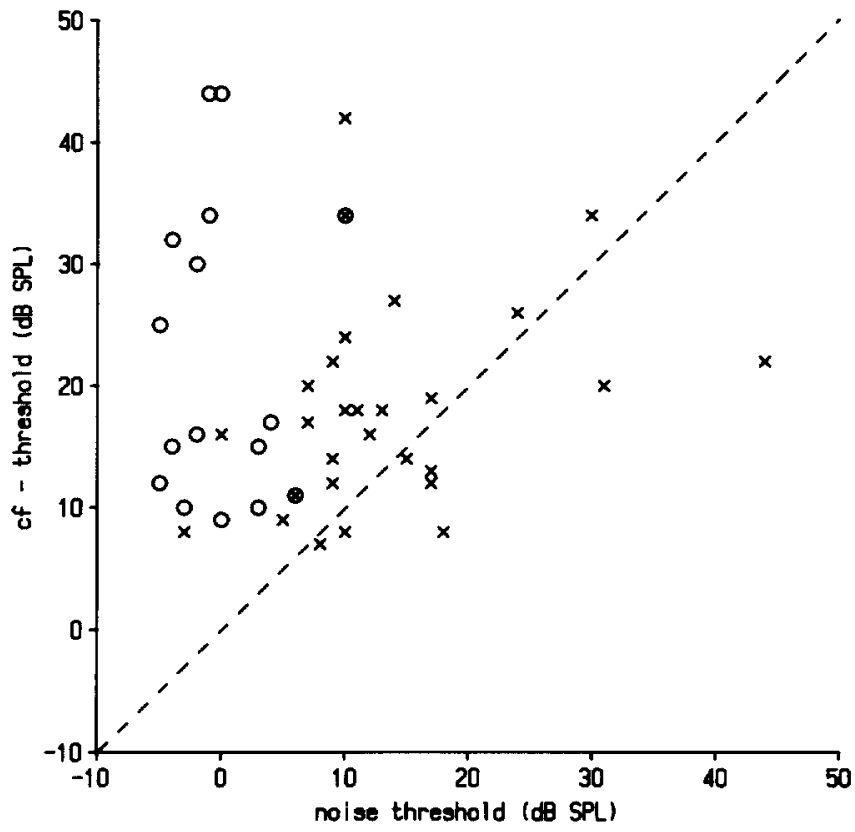

Fig. 6. Correlation between pure tone threshold and noise threshold in units responding to faint human respiration sounds (circles, $n=16$ ) and units not responding to this stimulus (crosses, $n=28$ ). The $45^{\circ}$-line indicates threshold values equal for pure tone and noise stimuli
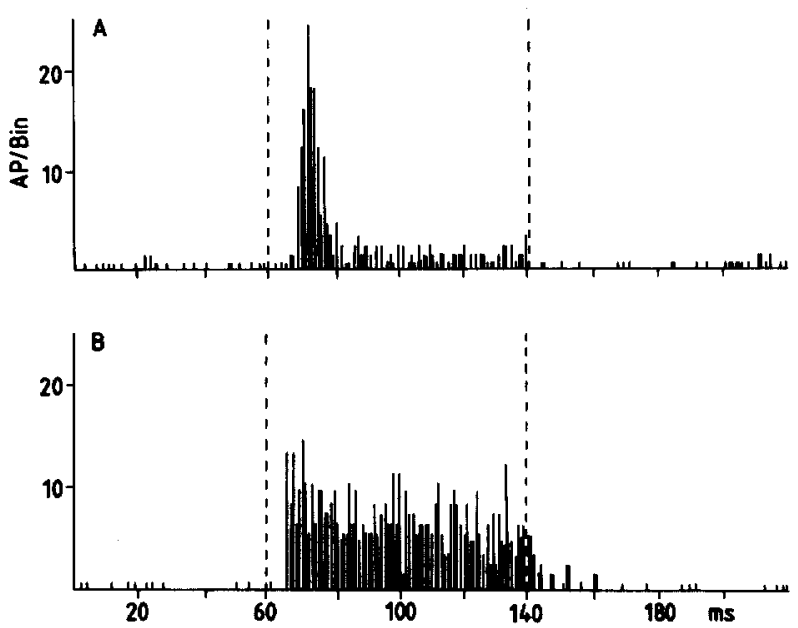

Fig. 7A, B. PST-histogram (bin width $1 \mathrm{~ms}$ ) of noise-unit type $\mathrm{I}$. Stimulus: bandpassed noise $26.7 \pm 10 \mathrm{kHz}$ (threshold value $0 \mathrm{~dB}$ SPL); A $10 \mathrm{~dB}$ above threshold, $\mathrm{B} 40 \mathrm{~dB}$ above threshold

sity range and had distinct upper thresholds. In three spontaneously active neurons excitation was only recorded in the threshold range, and activity was totally inhibited by noise stimuli $20 \mathrm{~dB}$ above threshold (Fig. 9A, B). One non-spontaneously active unit responded only in the intensity range between 4 and $19 \mathrm{~dB}$ SPL (Fig. 9C, D). Since these specialists for faint noises were difficult to detect, we suspect that this category may be represented in the rostro-medial IC in larger numbers than indicated by our sample.

In various parts of the IC we additionally recorded 59 units which were sensitive to bandpass filtered noise
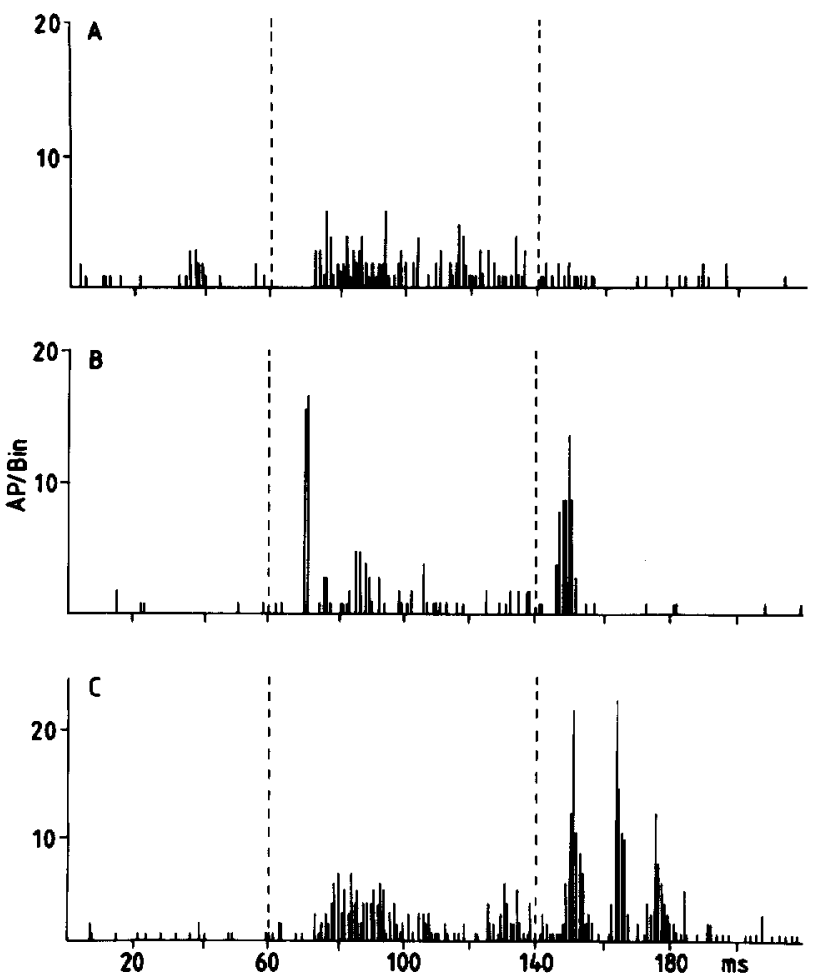

Fig. 8A-C. Noise-unit type II. Stimulus: bandpassed noise $22.5 \pm 10 \mathrm{kHz}$ (threshold value $-3 \mathrm{~dB}$ SPL); $\mathrm{A} 4 \mathrm{~dB}$ above threshold, B $20 \mathrm{~dB}$ above threshold. C Bursting off-response of noise-unit type II to intense stimulation (bandpassed noise $21.8 \pm 10 \mathrm{kHz}, 50 \mathrm{~dB}$ above threshold; threshold value $8 \mathrm{~dB}$ SPL)

but did not react to breathing noise. Thresholds ranged between 0 and $55 \mathrm{~dB}$ SPL, and thus were considerably higher than those of units sensitive to breathing noise (Fig. 5B). Most of the units in this category were less sensitive to pure tones, but there was not as great a range of threshold differences as in units responding to breathing noise (Fig. 6). The BFs ranged from 1.5 to $100 \mathrm{kHz}$. Thus, in the range of BFs from 10 to $30 \mathrm{kHz}$ we found noise sensitive units which responded to breathing noise, and others which did not. Therefore, we conclude that sensitivity to breathing noise may be more specific than sensitivity to noise in general within the frequency band of 10 to $30 \mathrm{kHz}$.

\section{Discussion}

The most interesting result of this study is the detection of IC neurons which are sensitive to breathing noises. These units belong to a class of neurons, found in the dorsomedial part of the IC, which are highly sensitive to noise bands in the frequency range below that of the echolocation signals emitted by Desmodus rotundus. A few of these units vigorously responded to breathing noises but not to noise signals of various center frequencies and bandwidths. We therefore suspect that these units are optimally activated by some as yet undefined stimulus parameters of breathing noise. The data suggest that some of the breathing-sensitive units are optimally 

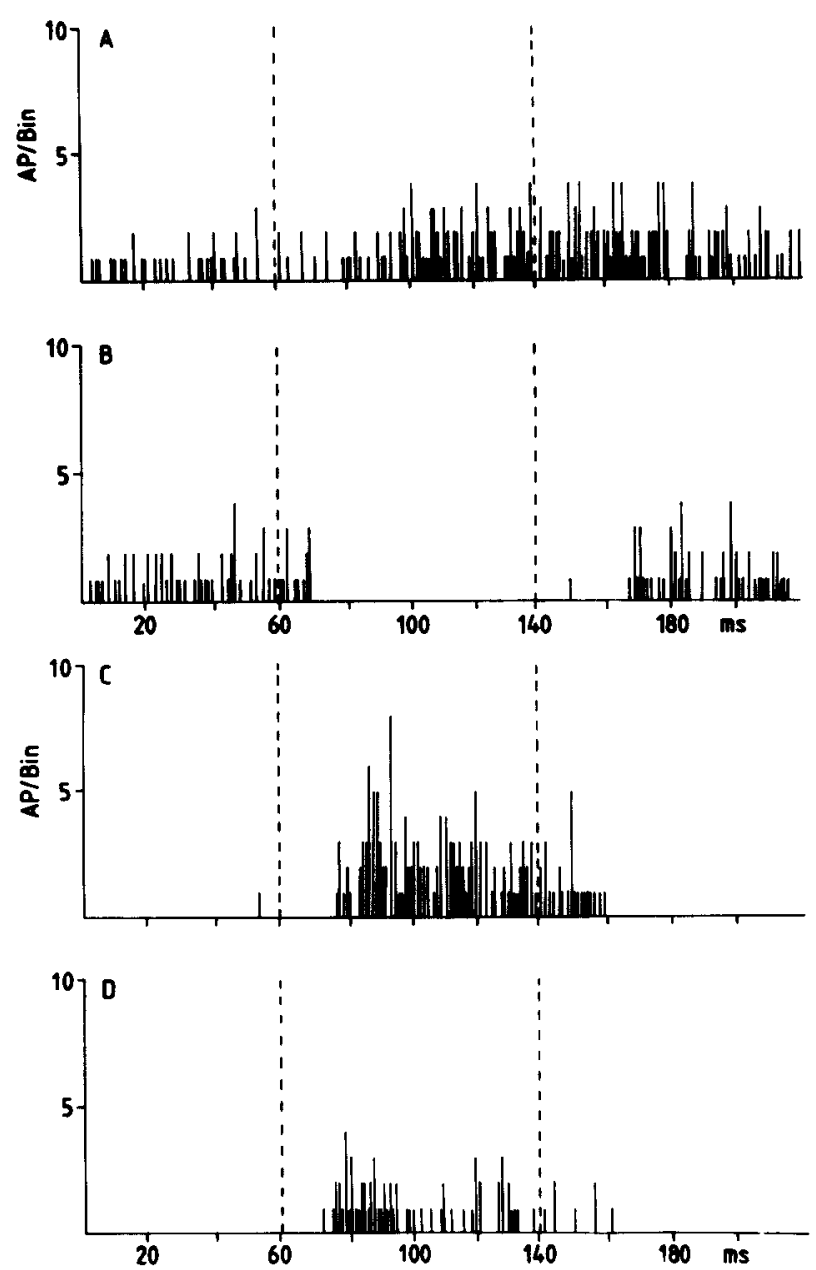

Fig. 9A-D. Noise-unit type III (spontaneously active neuron). Stimulus: bandpassed noise $21.5 \pm 10 \mathrm{kHz} ; \mathbf{A}$ threshold intensity $(8 \mathrm{~dB}$ SPL), B $20 \mathrm{~dB}$ above threshold. C/D Noise-unit type III (not spontaneously active). Stimulus: bandpassed noise $29 \pm 10 \mathrm{kHz}$ (threshold value $4 \mathrm{~dB}$ SPL); C $5 \mathrm{~dB}$ above threshold, $D 10 \mathrm{~dB}$ above threshold

activated by stimuli of long durations, however, the sample is still to small to make any definitive conclusion, and the units could not be held for a long enough time in order to investigate systematically the effect of all possible stimulus parameters.

In previous studies of the ascending auditory pathway, noise stimuli have mainly been used to evaluate signal/noise ratios or for system analysis in terms of information theory (e.g. Møller 1982). Evans and Whitfield (1964) were among the first who considered noise pulses as a class of natural auditory stimuli: In the auditory cortex of cats $27 \%$ of 125 units, they studied, responded only to clicks and odd sounds such as jangling of keys. Such units habituated easily. Specificity for odd sounds was considered to be a cortical feature not to be expected in subcortical nuclei. However, Suga (1969) recorded 3 noise-specific units among 250 neurons of the IC in two echolocating bat species. Since noise sensitivity did not make much sense in the context of echolocation, little attention has been paid to this early discovery. In the false vampire bat, Megaderma lyra, Rübsamen et al. (1988) described IC neurons which were extremely sensitive to faint noises and odd sounds such as brushing of the experimenter's beard or clothing. Megaderma lyra is known to detect ground-dwelling prey (e.g. frogs, mice) by passive detection of rustling noises and not by echolocation (Fiedler 1979; Marimuthu and Neuweiler 1987). Therefore, such units sensitive to faint noises may be interpreted as an adaptation to the auditory detection of concealed prey (Neuweiler 1990). It is tempting to interpret the IC units sensitive to breathing noises as a specific adaptation for the detection of resting mammals by vampire bats. However, since virtually nothing is known about how vampire bats search for and find wild prey or live-stock (Schmidt 1988), such speculations are premature. Our results suggest that prey-generated noises should be included in the list of possible clues vampire bats might use for prey detection and location.

This suggestion is corroborated by the audiogram and the IC tonotopy described in this study. The vampire bat is most sensitive in the frequency range between 10 and $20 \mathrm{kHz}$, with thresholds around $-10 \mathrm{~dB}$ SPL. This is clearly below the frequency band used for echolocation, even if one includes the faint fundamental. Desmodus rotundus emits brief frequency modulated and multiharmonic echolocation signals of 0.4 to $1.4 \mathrm{~ms}$ duration. The main energy of the sound is in the frequency range of the second and the third harmonic which sweep from about 75 to $45 \mathrm{kHz}$, respectively, from about 100 to $65 \mathrm{kHz}$. The fundamental that sweeps from about 35 to $25 \mathrm{kHz}$ seems to be present during flight, but is 20 to $25 \mathrm{~dB}$ less intense than the other harmonics (Joermann and Schmidt 1981).

The neuronal audiogram derived from the IC recordings in awake specimens described here is distinctly different from the ones recorded by cochlear microphonic potentials in anesthetized vampire bats (Vernon and Peterson 1966). In the latter study, the lowest thresholds occurred at $50-60 \mathrm{kHz}$ and increased monotonically by nearly $1 \mathrm{~dB} / 1 \mathrm{kHz}$ for lower frequencies. This discrepancy may be explained by the effects of anesthesia. In 16 species of echolocating bats in which audiograms have been recorded under anesthesia thresholds are generally high with a minimum in the frequency range of the emitted echolocation sounds. However, in 24 different species recorded under awake conditions, the audiograms uniformly have much lower thresholds and all species are most sensitive in the low frequency range between 12 and $25 \mathrm{kHz}$, and usually less sensitive by a few $\mathrm{dB}$ up to $20 \mathrm{~dB}$ in the ultrasonic frequency range of their echolocation signals (Neuweiler 1990). Obviously, the vampire bat may be added as the 25 th species to this list. These uniform data from many different species and from various labs indicate that it is common for bats to be highly sensitive to lower frequencies which are prominent in the power spectra of all sorts of noises generated by animals. Therefore, all echolocating bats may sensitively listen to such noises and be attracted to or repelled from locations from which such noises emanate. The vampire bats (this study) and the false vampires (Rübsamen et al. 1988; Guppy and Coles 1988) might 
simply be outstanding examples of a general auditory sensitivity to faint noises in all bats. For instance, the insectivorous bat Antrozous pallidus mainly detects its prey by sound of its flight and other movement and not by echolocation (Bell 1982). Other echolocating bats are attracted to insect aggregations by their humming flight noises (Buchler and Childs 1981).

In vampire bats, high sensitivity to lower frequencies coincides with a good representation of frequencies up to $35 \mathrm{kHz}$ in the dorsal and lateral parts of the IC. However, frequencies from about 50 to $100 \mathrm{kHz}$ are equally well represented in the ventral half of the IC. Only the representation of the frequency band in between these two large portions, from 40 to about $50 \mathrm{kHz}$, is squeezed into a thin sheet. The same sort of underrepresentation has been observed in Hipposideros speoris. This bat emits echolocation signals within the frequency range of 110 and $140 \mathrm{kHz}$, and the frequency band from 110 to $75 \mathrm{kHz}$ is compressed into a thin sheet within the tonotopy of the IC (Rübsamen et al. 1988). In the horseshoe bat Rhinolophus rouxi, which emits sounds from about 65 to $78 \mathrm{kHz}$, the frequencies of 60 to $40 \mathrm{kHz}$ are poorly represented (unpubl. data). Thus, it appears that in several bat species the transitional area between echolocation and passive listening is poorly represented in the tonotopy of the ascending auditory pathway.

The results from various species of bats suggest that a detailed comparative study of tonotopy will disclose those frequency bands which are behaviorally significant. This is especially obvious in the tonotopy of horseshoe bats and hipposiderids which is focused not only on the species-specific frequency band of echolocation, but also precisely on the frequency of a given individual. In the vampire bat the specific tonotopy suggests that not only echolocation signals, but also auditory events at low frequencies are highly significant.

Scanning through the literature on audition in mammals quickly discloses that the general rule of a logarithmic frequency representation is modified in most cases by overrepresentations of specific frequency bands. In mice the largest percent volume of the IC is occupied by neurons with BFs between 15 and $35 \mathrm{kHz}$ (Stiebler and Ehret 1985). In cats the frequency range from 5 to $30 \mathrm{kHz}$ is overrepresented in the IC (Rockel and Jones 1973), and in the rabbit low frequencies up to $6 \mathrm{kHz}$ occupy the dorsal half of the IC (Aitkin el al. 1972). In the marsupial Dasyurus hallucatus an overrepresentation from 10 to $40 \mathrm{kHz}$ coincides with lowest thresholds of $-10 \mathrm{~dB}$ SPL in the same frequency range (Aitkin et al. 1986). Apparently overrepresentation of specific frequency bands is not just a specialization restricted to echolocating bats, but is rather the rule in mammals.

Noise sensitive neurons may be specific adaptations for species which have to detect and locate prey acoustically; our results suggest that vampire bats belong into this category of mammals.

Acknowledgements. We want to thank Dr. Christel Schmidt for her assistance in the experiments, and Dr. Ellen Covey for correcting the English. This study was supported by VDFG, SFB 203, Gehör, München.

\section{References}

Aitkin LM, Fryman S, Blake DW, Webster WR (1972) Responses of neurones in the rabbit inferior colliculus. I. Frequency specificity and topographic arrangement. Brain Res 47:77-90

Aitkin LM, Irvine DRF, Nelson JE, Merzenich MM, Clarey JC (1986) Frequency representation in the auditory midbrain and forebrain of a marsupial, the northern native cat, Dasiurus hallucatus. Brain Behav Evol 29:17-28

Bell GP (1982) Behavioral and ecological aspects of gleaning by the desert insectivorous bat Antrozous pallidus (Chiroptera: Vespertilionidae). Behav Ecol Sociobiol 10:217-223

Buchler ER, Childs SB (1981) Orientation to distant sounds by foraging big brown bats (Eptesicus fuscus). Anim Behav 29:428-432

Evans EF, Whitfield IC (1964) Classification of unit responses in the auditory cortex of the unanesthetized and unrestrained cat. J Physiol 179:476-493

Fiedler J (1979) Prey catching with and without echolocation in the Indian false vampire bat (Megaderma lyra). Behav Ecol Sociobiol $6: 155-160$

Greenhall AM, Schmidt U (eds) (1988) Natural history of vampire bats. CRC Press, Boca Raton

Guppy A, Coles RB (1988) Acoustical and neural aspects of hearing in the Australian gleaning bats, Macroderma gigas and $\mathrm{NyC}$ tophilus gouldi. J Comp Physiol A 162:653-668

Joermann G, Schmidt U (1981) Echoortung bei der Vampirfledermaus, Desmodus rotundus. II. Lautaussendung im Flug und Korrelation zum Flügelschlag. Z Säugetierkunde 46:136-146

Marimuthu G, Neuweiler G (1987) The use of acoustical cues for prey detection by the Indian false vampire bat, Megaderma lyra. J Comp Physiol A 166:509-515

Møller A (1982) Use of pseudorandom noise in studies of the dynamic properties of the linear part of a sensory neural system. Proc 15th Inter Conf Syst Sci 2:337-351

Neuweiler G (1990) Auditory adaptations for prey capture in echolocating bats. Physiol Rev 70:615-641

Payne RS (1971) Acoustic location of prey by barn owls. J Exp Biol $54: 535-573$

Pollak GD, Schuller G (1981) Tonotopic organization and encoding features of single units in the inferior colliculus of horseshoe bats: functional implications for prey identification. J Neurophysiol 45:208-226

Rockel AJ, Jones EG (1973) The neuronal organization of the inferior colliculus of the adult cat. I. The central nucleus. J Comp Neurol 147:11-60

Rübsamen R, Neuweiler G, Sripathi K (1988) Comparative collicular tonotopy in two bat species adapted to movement detection, Hipposideros speoris and Megaderma lyra. J Comp Physiol A $163: 271-285$

Schlegel P, Jen P, Singh S (1988) Auditory spatial sensitivity of inferior collicular neurons of echolocating bats. Brain Res $456: 127-138$

Schmidt U (1978) Vampirfledermäuse. A. Ziemsen, Wittenberg Lutherstadt

Schmidt U (1988) Orientation and sensory functions in Desmodus rotundus. In: Greenhall AM, Schmidt U (eds) Natural history of vampire bats. CRC Press, Boca Raton, pp 143-166

Schnitzler H-U, Menne D, Kober R, Heblich K (1983) The acoustical image of fluttering insects in echolocating bats. In: Huber $F$, Markl H (eds) Neuroethology and behavioral physiology. Roots and growing points. Springer, Berlin, Heidelberg, New York, pp 235-250

Stiebler I, Ehret G (1985) Inferior colliculus of the house mouse. I. A quantitative study of tonotopic organization, frequency representation, and tone-threshold distribution. J Comp Neurol $238: 65-76$

Suga N (1969) Classification of inferior collicular neurones of bats in terms of responses to pure tones, FM sounds and noise bursts. J Physiol 200:555-574

Vernon J, Peterson E (1966) Hearing in the vampire bats, Desmodus rotundus murinus, as shown by cochlear potentials. J Audit Res $6: 181-187$ 